\title{
The Relationship Between Neutrophil- Lymphocyte and Platelet-Lymphocyte Ratios With Hospital Stays and Mortality in the Emergency Department
}

\author{
Mustafa Cifci ${ }^{1}$, Huseyin C. Halhalli ${ }^{1}$ \\ 1. Emergency Medicine, University of Health Sciences, Kocaeli Derince Training and Research Hospital, Kocaeli, TUR
}

Corresponding author: Huseyin C. Halhalli, dr.cahithalhalli@gmail.com

\section{Abstract \\ Background}

Most acute pancreatitis scoring is made in the first 48-72 hours or later. Like many inflammatory processes, Neutrophil/ymphocyte ratio (NLR) and platelet/lymphocyte ratio (PLR) can be useful in showing the severity and extent of inflammation in acute pancreatitis. Our study aimed to evaluate whether these rates affect mortality according to the NLR and PLR values of patients diagnosed with acute pancreatitis by examining the blood samples taken within the first hour after admission to the emergency department rates are useful in predicting the length of stay.

\section{Methods}

In our retrospective study, 557 patients applied to our clinic for 4.5 years, whose amylase and lipase values were higher than two times the cut-off value in blood tests and whose CT imaging was compatible with acute pancreatitis were included in the study.

\section{Results}

The median length of hospitalization of the patients was 4.0 (3.0-6.0) days. Gallstones were detected in 320 (57.5\%) patients. Mortality of less than a year was observed in 45 (8.1\%) of the study population. Eighteen of the patients (3.2\%) showed the need for follow-up in the intensive care unit. A statistically significant relationship was found between mortality and variables hematocrit (HCT), red cell distribution width (RDW), c-reactive protein CRP), glucose, urea, potassium, albumin, PLR, and NLR ( $\mathrm{p}<0.05)$. A statistically significant correlation was observed between RDW, NLR, glucose, and CRP levels in the two groups divided according to the median value of 4 days we found on hospitalization $(\mathrm{p}<0.05)$. According to the graphics and test results obtained by ROC analysis, the mortality status can be predicted at a statistically significant level with PLR and NLR diagnostic tests $(\mathrm{p}<0.05)$.

Review began $12 / 03 / 2020$ Review ended 12/19/2020 Published 12/20/2020

\section{() Copyright 2020}

Cifci et al. This is an open access article distributed under the terms of the Creative Commons Attribution License CC-BY 4.0., which permits unrestricted use, distribution, and reproduction in any medium, provided the original author and source are credited.

\section{Conclusion}

High levels of NLR, PLR, RDW, glucose, CRP, urea, potassium, low albumin and hematocrit values at the first admission in the Emergency Service seem to be associated with increased 1-year mortality in acute pancreatitis.

Categories: Emergency Medicine, Gastroenterology, Hematology

Keywords: acute pancreatitis, neutrophil/lymphocyte ratios, platelet/lymphocyte ratios

\section{Introduction}

Acute pancreatitis is an inflammatory process with mortality varying between $3 \%$ and $17 \%$ [1,2]. Because of these high mortality rates, different scoring systems have been developed based on clinical and laboratory findings, radiological risk factors, severity grading systems, and various serum markers [3]. While some of these scorings are done when the patient is admitted immediately to help the patient's triage, some are evaluated in the first 48-72 hours or later [4]. While the sensitivity is between $40-90 \%$ in the Ranson Scoring System, 56\% sensitivity was observed in BISAP (Bedside Index for Severity in Acute Pancreatitis) $[5,6]$. The Modified Glasgow 2 scoring has a 56-85\% sensitivity in evaluating the severity of both alcohol-related and biliary pancreatitis [7]. Even if all these scoring systems' sensitivity and specificity in predicting the severity of acute pancreatitis vary between $55 \%$ and $90 \%$ according to the cut-off values of the parameters used and the scoring timing, these scoring must complete at least 48 hours to apply. Also, the complexity of the scoring limits their use [8].

Neutrophil/lymphocyte ratio (NLR) and platelet/lymphocyte ratio (PLR) have been accepted as useful indicators to predict prognosis and survival in various cancers that show inflammatory processes [9]. Like 
many inflammatory processes, NLR and PLR can be useful in showing the severity and extent of inflammation in acute pancreatitis. Since the severity of the disease in acute pancreatitis is related to the inflamed pancreatic tissue size, the NLR and PLR ratios may also be useful in demonstrating this relationship. In our study, we aimed to evaluate whether these rates affect mortality according to NLR and PLR values of patients diagnosed with acute pancreatitis by examining the blood samples taken within the first hour after admission to the emergency department and whether these rates are useful in predicting the length of stay.

\section{Materials And Methods Study population}

Our study was carried out in a 3rd level academic emergency medicine clinic to which approximately 400000 patients apply annually. Our study was conducted among patients diagnosed with acute pancreatitis after admission to the emergency department.

\section{Study design}

Our study is a retrospective study. By examining our hospital's computer-based data system, patients who applied to our clinic for 4.5 years between 01.01.2015-01.07.2019, amylase and lipase values higher than two times the cut-off value in blood tests and CT imaging compatible with acute pancreatitis were detected. Demographic data (age, gender), blood amylase, lipase, glucose, albumin, lactate dehydrogenase (LDH), aspartate aminotransferase (AST), alanine aminotransferase (ALT), c-reactive protein (CRP), urea, creatinine (CRE), sodium (Na), potassium (K), chlorine (Cl), calcium (Ca) levels, haemoglobin in the blood (HGB), hematocrit (HCT), erythrocyte count (RBC), mean platelet volume (MPV), erythrocyte distribution range (RDW), leukocyte (LEU), neutrophil (NEU), platelet (PLT), lymphocyte (LNF) counts, Neutrophil / Lymphocyte count ratio (NLR), Platelet / Lymphocyte count ratio (PLR) were determined. Besides, the duration of hospital stay, intensive care needs, bile ducts and gallstones, and one-year mortality after diagnosis were determined. Patients who did not have sufficient information in the data system (with or without blood tests or imaging findings) left the hospital voluntarily, patients with haematological malignancies referred to other hospitals, and patients under the age of 18 were excluded from the study. Only the first applications of the patients with more than one application were evaluated.

\section{Statistical analysis}

The data's compliance with normal distribution was evaluated by a histogram, q-q graphs, and Shapiro-Wilk test. Variance homogeneity was tested with the Levene test. In comparison between paired groups, an independent two-sample t-test was used for quantitative variables. The normality assumption was provided, and Mann Whitney U test was used for those who did not provide normality assumption. Chi-square analysis was used to compare categorical variables. ROC charts were obtained by applying ROC (Receiver Operating Characteristic) analysis to evaluate the diagnostic test performance in determining the mortality status of Platelet / Lymphocyte and Neutrophil / Lymphocyte diagnostic tests. Sensitivity, selectivity, positive predictive value, and negative predictive values were calculated for the cut-off values determined according to the Youden method. The data analysis was carried out in TURCOSA (Turcosa Analytics Ltd Co, Turkey, www.turcosa.com.tr) statistics software. The significance level was accepted as $\mathrm{p}<0.05$.

\section{Ethics committee approval}

The data collected were collected for study purposes only. Local Ethics Committee approval was obtained for the research on 12.03.2020 with the protocol number 2020-29.

\section{Results}

Five hundred eighty-nine patients were diagnosed with acute pancreatitis after admission to the emergency service between 01.01.2015 and 01.07.2019. Five hundred fifty-seven of these were included in the study. Sixteen patients left the hospital voluntarily, 12 patients were transferred to another hospital, and four patients were excluded due to hematological malignancy diagnosis. The average age of 557 observations obtained in the study was $58.66 \pm 17.30 ; 315$ of them (56.6\%) were women. The patients' median length of hospitalization was found to be 4.0 (3.0-6.0) days. Gallstones were detected in 320 (57.5\%) patients. Mortality of less than one year was observed in 45 (8.1\%) of the study population. Eighteen (3.2\%) of the patients showed the need for follow-up in the intensive care unit. The median of Ranson score of observations in the study was 1.0 (1.0-2.0), and the Ranson score of 131 (23.5\%) was three and above (Table 1). Laboratory variables related to observations are shown in Table 1 . The median, first, and third quartile values for PLR and NLR were 156.7 (103.2-223) and 4.6 (2.8-8.2), respectively (Table 1). 


\section{Cureus}

\begin{tabular}{|c|c|c|}
\hline & Mean & SD \\
\hline Age (Years) & 58.66 & 17.30 \\
\hline Garden (Male, \%) & $242(\% 43.4)$ & \\
\hline Total Hospital Stay (Day) & 4.0 & 4.16 \\
\hline ICU Stay (Day) Yes No & 18 (\%3.2) 539 (\%96.8) & \\
\hline Gallstone (\%) Yes No & 320 (\%57.5) 237 (\%42.5) & \\
\hline Mortality (\%) $\square 1$ year < 1 year & $512(\% 91.9) 45(\% 8.1)$ & \\
\hline RANSON Skore <3 प3 & $426(\% 76.5) 131(\% 23.5)$ & \\
\hline \multicolumn{3}{|l|}{ Laboratory Data } \\
\hline Glucose (mg/dL) & 127.0 & 66.16 \\
\hline Urea (mg/dL) & 29.0 & 25.39 \\
\hline Creatinine (mg/dL) & 0.9 & 0.86 \\
\hline ALT (U/L) & 99.0 & 182.61 \\
\hline AST (U/L) & 121.0 & 210.50 \\
\hline LDH (U/L) & 313.0 & 305.56 \\
\hline Albumin (g/dL) & 4.02 & 0.39 \\
\hline Amylase (U/L) & 912.0 & 1365.49 \\
\hline Lipase (U/L) & 1672.0 & 3313.35 \\
\hline $\mathrm{Na}(\mathrm{mEq} / \mathrm{L})$ & 138.71 & 3.27 \\
\hline K (mEq/L) & 4.10 & 0.42 \\
\hline CPR (mg/dL) & 6.8 & 40.61 \\
\hline WBC (느) & 10687.97 & 4179.04 \\
\hline RBC $\left(10^{6} \underline{L}\right.$ L) & 4.33 & 0.56 \\
\hline HCT (\%) & 38.96 & 4.64 \\
\hline RDW (\%) & 15.75 & 2.22 \\
\hline Platelets (느) & 246544.34 & 82606.56 \\
\hline Neutrophil (느) & 7400.0 & 3975.25 \\
\hline Lymphocyte(e L) & 1500.0 & 1326.34 \\
\hline Platelets/lymphocyte ratio & 156.7 & 149.25 \\
\hline Neutrophile/lymphocyte ratio & 4.6 & 7.72 \\
\hline
\end{tabular}

\section{TABLE 1: Baseline characteristics of the patients with acute pancreatitis}

ICU, intensive care unit; ALT, alanine aminotransferase; AST, aspartate aminotransferase; LDH, lactate dehydrogenase; Na, Sodium; K, Potassium; $\mathrm{Ca}$, calcium; CRP, C reactive protein; WBC, white blood cell; RBC, red blood cell; Hct; Hematocrit; RDW. red cell distribution width; SD, standard deviation.

Age, urea, AST, ALT, LDH, NEU, LNF, NLR, and PLR values were compared in the data divided into two groups according to the Ranson score received at the first admission to the emergency service. As a result of these comparisons, age, urea, AST, ALT, LDH, NEU, LNF, PLR, and NRL variables were found to be statistically significantly different in the groups with Ranson score below three and above $3(p<0.05)$ (Table 2). 


\section{Cureus}

\begin{tabular}{|c|c|c|c|}
\hline & \multicolumn{2}{|l|}{ RANSON } & \multirow{3}{*}{$\mathbf{p}$} \\
\hline & RANSON $<3$ & RANSON $\geq 3$ & \\
\hline & $(n=426)$ & $(n=131)$ & \\
\hline Age (Years) & $55.65 \pm 17.47$ & $68.45 \pm 12.47$ & $<0.001$ \\
\hline Urea (mg/dL) & $29.0(25.0-33.0)$ & $31.0(27.0-45.0)$ & 0.002 \\
\hline AST (U/L) & 79.0(27.0-173.0) & $362.0(264.0-518.0)$ & $<0.001$ \\
\hline ALT (U/L) & $60.0(22.0-168.0)$ & $250.0(159.0-406.0)$ & $<0.001$ \\
\hline LDH (U/L) & $276.5(219.0-355.0)$ & $530.0(424.0-655.0)$ & $<0.001$ \\
\hline Neutrophil ( $($ L) & $7000.0(5000.0-9400.0)$ & $8600.0(6000.0-12700.0)$ & $<0.001$ \\
\hline Lymphocyte (느) & $1600.0(1200.0-2300.0)$ & $1300.0(800.0-2000.0)$ & $<0.001$ \\
\hline Platelets/lymphocyte ratio & 151.8(98.3-213.0) & 190.5(119.4-275.7) & $<0.001$ \\
\hline Neutrophile/lymphocyte ratio & $4.3(2.5-7.1)$ & $6.8(3.7-11.7)$ & 0.001 \\
\hline
\end{tabular}

\section{TABLE 2: Comparison of patients with Ranson score $<3$ vs. patients with Ranson score $\geq 3$}

AST, aspartate aminotransferase; ALT, alanine aminotransferase; LDH, lactate dehydrogenase.

Mortality was similar according to gender distribution, and there was no statistically significant difference ( $p>0.05$ ). No statistically significant relationship was found between the groups formed according to the mortality time and the variables of emergency admission Ranson score, WBC, RBC, MPV, PLT, NEU, LNF, Na, AST, ALT, LDH, CRE, Amylase, Lipase ( $p>0.05$ ). There was no statistically significant difference between the presence of obstruction and mortality $(p>0.05)$.

A statistically significant correlation was found between mortality and variables HCT, RDW, CRP, glucose, urea, potassium, albumin, PLR, and NLR ( $\mathrm{p}<0.05$ ) (Table 3). A statistically significant relationship was observed between the need for intensive care hospitalization and PLR, NLR, Glucose, CRP, and Albumin levels $(\mathrm{p}<0.05)$ (Table 4). A statistically significant correlation was observed between RDW, NLR, glucose, and CRP levels in the two groups that we divided according to the median value of 4 days, which we found on hospitalization $(\mathrm{p}<0.05)$ (Table 4$)$. 


\section{Cureus}

\begin{tabular}{|c|c|c|c|}
\hline & \multicolumn{3}{|l|}{ Mortality } \\
\hline & $>1$ years $(n=512)$ & $\leq 1$ years $(n=45)$ & \\
\hline Age (Years) & $57.39 \pm 16.9$ & $73.04 \pm 15.33$ & $<0.001$ \\
\hline Garden (Male, \%) & 224(43.8) & 18(40.0) & 0.627 \\
\hline Hospital Stay (Day) & $4.0(3.0-6.0)$ & $6.0(4.0-11.0)$ & $<0.001$ \\
\hline \multicolumn{4}{|l|}{ ICU Stay } \\
\hline Yes & $4(0.8)$ & $14(31.1)$ & \multirow[t]{2}{*}{$<0.001$} \\
\hline No & 508(99.2) & $31(68.9)$ & \\
\hline \multicolumn{4}{|l|}{ Gallstone } \\
\hline No & 216(42.2) & $21(46.7)$ & \multirow[t]{2}{*}{0.560} \\
\hline Yes & 296(57.8) & 24(53.3) & \\
\hline \multicolumn{4}{|l|}{ RANSON Score } \\
\hline$<3$ & $397(77.5)$ & $29(64.4)$ & \multirow[t]{2}{*}{0.071} \\
\hline$\geq 3$ & 115(22.5) & 16(35.6) & \\
\hline \multicolumn{4}{|l|}{ Laboratory Data } \\
\hline Glucose (mg/dL) & 126.5(108-153.5) & $151(117-221)$ & $<0.001$ \\
\hline Urea (mg/dL) & $29.0(25.0-33.0)$ & $41.0(26.0-60.0)$ & $<0.001$ \\
\hline Albumin (g/dL) & $4.05 \pm 0.35$ & $3.67 \pm 0.60$ & $<0.001$ \\
\hline CPR (mg/dL) & $6.3(2.0-25.6)$ & $12.4(6.3-50.7)$ & 0.002 \\
\hline НCT (\%) & $39.1 \pm 4.54$ & $37.33 \pm 5.49$ & 0.041 \\
\hline RDW (\%) & $15.66 \pm 2.10$ & $16.79 \pm 3.11$ & 0.001 \\
\hline Platelets (느) & $246984.38 \pm 83920.62$ & $241537.78 \pm 66405.41$ & 0.672 \\
\hline Neutrophil ( $(\underline{L}$ ) & 7400.0(5200.0-9900.0) & $7900.0(6200.0-11200.0)$ & 0.090 \\
\hline Lymphocyte(느) & $1600.0(1100.0-2300.0)$ & $1200.0(800.0-1600.0)$ & 0.063 \\
\hline Platelets/lymphocyte ratio & 154.7(101.2-214.9) & $227.0(129.0-295.7)$ & 0.004 \\
\hline Neutrophile/lymphocyte ratio & $4.5(2.7-7.9)$ & $6.6(3.8-11.5)$ & 0.004 \\
\hline
\end{tabular}

TABLE 3: Comparison of patients with mortality

ICU, intensive care unit; CRP, C reactive protein; Hct; Hematocrit; RDW. red cell distribution width; SD, standard deviation. 


\section{Cureus}

\begin{tabular}{|c|c|c|c|c|c|c|c|c|c|c|}
\hline & \multicolumn{5}{|c|}{ Hospital Stay (Day) } & \multicolumn{5}{|c|}{ ICU Stay (Day) } \\
\hline & \multicolumn{2}{|c|}{$\leq 4$ day $(n=300)$} & \multicolumn{2}{|c|}{$>4$ day $(n=227)$} & \multirow[t]{2}{*}{$\mathrm{p}$} & \multicolumn{2}{|l|}{ Yes $(n=18)$} & \multicolumn{2}{|c|}{ No $(n=539)$} & \multirow[t]{2}{*}{$\mathrm{p}$} \\
\hline & Mean & SD & Mean & SD & & Mean & SD & Mean & SD & \\
\hline Age (Years) & 57.24 & 17.74 & 60.32 & 16.65 & 0.036 & 63.44 & 21.56 & 58.5 & 17.14 & 0.290 \\
\hline Glucose (mg/dL) & 136.69 & 60.73 & 154.36 & 70.93 & 0.00005 & 214.61 & 112.04 & 142.51 & 62.92 & 0.010 \\
\hline Albumin (g/dL) & 4.04 & 0.31 & 4.00 & 0.46 & 0.92104 & 3.53 & 0.76 & 4.03 & 0.36 & 0.010 \\
\hline Amylase (U/L) & 1338.48 & 1206.66 & 1505.27 & 1527.72 & 0.36047 & 1032.17 & 1487.66 & 1428.24 & 1360.85 & 0.07 \\
\hline Lipase (U/L) & 3367.70 & 3151.73 & 3549.77 & 3496.23 & 0.84796 & 1939.17 & 2067.24 & 3502.22 & 3336.38 & 0.421 \\
\hline $\mathrm{CPR}(\mathrm{mg} / \mathrm{dL})$ & 17.94 & 28.41 & 31.23 & 50.40 & 0.00017 & 60.67 & 87.58 & 22.86 & 37.62 & 0.026 \\
\hline RDW (\%) & 15.59 & 2.35 & 15.94 & 2.05 & 0.01881 & 15.83 & 1.77 & 15.75 & 2.23 & 0.726 \\
\hline Neutrophil (느) & 7659.33 & 3568.08 & 8594.16 & 4356.50 & 0.02094 & 10005.56 & 6065.28 & 8026.72 & 3878.41 & 0.214 \\
\hline Lymphocyte (QL) & 1840.00 & 1105.05 & 1750.97 & 1546.02 & 0.09462 & 1561.11 & 1382.3 & 1806.86 & 1325.03 & 0.052 \\
\hline Platelets/lymphocyte ratio & 176.63 & 120.42 & 207.94 & 175.83 & 0.05951 & 226.06 & 111.23 & 189.91 & 150.3 & 0.042 \\
\hline Neutrophile/lymphocyte ratio & 6.14 & 6.30 & 7.79 & 9.03 & 0.00427 & 9.9 & 7.38 & 6.8 & 7.72 & 0.024 \\
\hline
\end{tabular}

\section{TABLE 4: Comparison of patients with hospitality}

CRP, C reactive protein; RDW, red cell distribution width; SD, standard deviation.

According to the graphics and test results obtained by ROC analysis, mortality can be predicted at a statistically significant level with PLR and NLR diagnostic tests $(\mathrm{p}<0.05)$ (Figure 1 ).

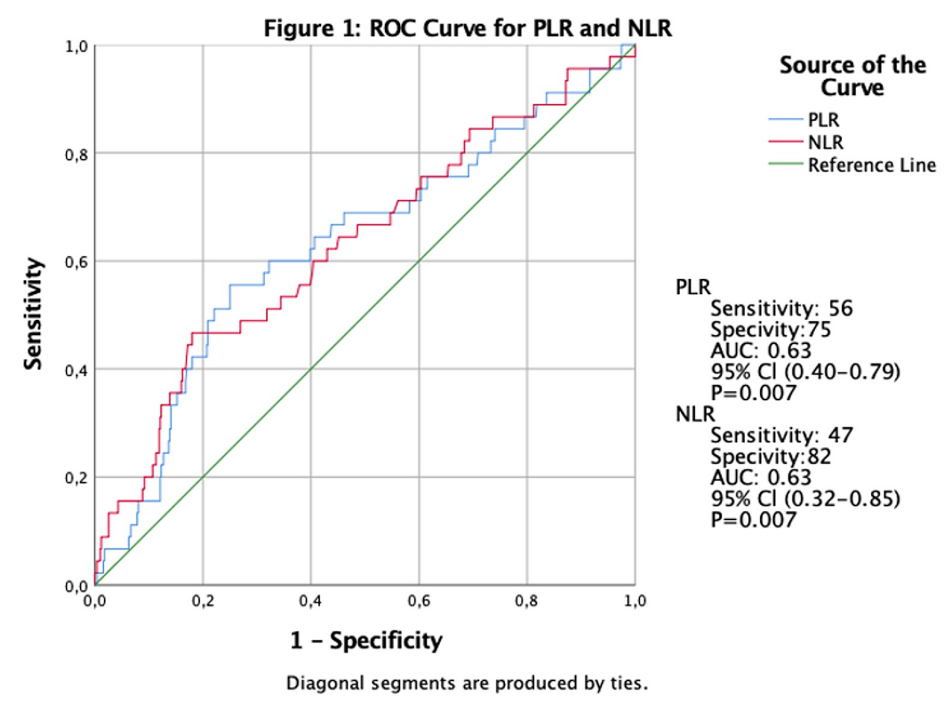

FIGURE 1: ROC curve for PLR and NLR for one-year mortality in patients with acute pancreatitis

PLR: Platelet / Lymphocyte count ratio, NLR: Neutrophil / Lymphocyte count ratio, AUC: Area under the curve, ROC: Receiver operating characteristic, Cl: Confidence interval)

Performance criteria obtained according to the cut-off value determined for Platelet / Lymphocyte (215) are given in Table 5. The probability of the observation with a mortality period of 1 year or less from two randomly selected observations according to the platelet/lymphocyte ratio is one year or less according to the observation with a mortality duration of 1 year or more. The sensitivity of Platelet / Lymphocyte test; In 
the case of illness, the test's probability of giving a positive result is $56 \%$. Platelet / Lymphocyte test; In the absence of disease, there is a $75 \%$ chance that the test will give a negative result. Positive predictive value of Platelet / Lymphocyte test; If the test is positive, the probability of developing the disease is $16 \%$. Negative predictive value of the Platelet / Lymphocyte test; In cases where the test is negative, the probability of not developing the disease is $95 \%$. Performance criteria obtained according to the cut-off value ( $\geqslant 9.09$ ) determined for NLR are given in Table 5. The AUC value, according to the NLR at the cut-off value determined, is $62.8 \%$. The specificity of NLR was determined as $47 \%$, and its sensitivity was $82 \%$.

\begin{tabular}{|c|c|c|c|c|c|c|}
\hline \multirow{2}{*}{ Variables } & \multicolumn{4}{|c|}{ Statistical diagnostic measures } & \multicolumn{2}{|c|}{ ROC curve statistic } \\
\hline & SEN $(95 \% \mathrm{Cl})$ & SPE (95\%Cl) & PPV (95\%Cl) & NPV (95\%Cl) & AUC & $p$ \\
\hline Platelet / Lymphocyte (3215) & $0.56(0.40-0.70)$ & $0.75(0.71-0.79)$ & $0.16(0.14-0.27)$ & $0.95(0.91-0.96)$ & 0.628 & 0.007 \\
\hline Neutrophil / Lymphocyte (39.09) & $0.47(0.32-0.62)$ & $0.82(0.78-0.85)$ & $0.18(0.15-0.30)$ & $0.94(0.90-0.96)$ & 0.628 & 0.007 \\
\hline \multicolumn{7}{|c|}{$\begin{array}{l}\text { TABLE 5: Diagnostic test performance criteria obtained to determine mortality status with Platele } \\
\text { / Lymphocyte and Neutrophil / Lymphocyte diagnostic tests }\end{array}$} \\
\hline \multicolumn{7}{|c|}{$\begin{array}{l}\text { SEN, sensitivity; SPE, specificity; PPV, positive predictive value; NPV, negative predictive value; AUC, area under curve, ROC, receiver operating } \\
\text { characteristic, Cl, confidence interval }\end{array}$} \\
\hline
\end{tabular}

\section{Discussion}

Acute pancreatitis is an inflammatory disease with severe morbidity and mortality. The incidence of acute pancreatitis, which is currently diagnosed frequently in Emergency Departments, has increased over the last 25 years $[10,11]$. Also, diagnosis and treatment cause high costs [12]. For this reason, it may be useful to predict the severity of the disease and make risk stratification in Emergency Units with a large patient population. However, most scoring systems can be calculated 24 to 48 hours after hospitalization. Scoring systems, where the first blood tests taken in the emergency department can calculate the severity of the disease and the complications that may develop in a short time, can contribute to the provision of better care for patients with acute pancreatitis.

A statistically significant relationship was observed between NLR, PLR, RDW, and 28-day mortality in the study of Zhou et al., which included 472 patients in 2019 to predict acute pancreatitis disease severity and prognosis in the early period [13]. Although our study was conducted with a different population than this study and evaluated the 1 -year mortality, it has similarities with this study statistically. A statistically significant correlation was found in our study in terms of 1-year mortality in patients with increased NLR, PLR, and RDW levels $(\mathrm{p}<0.005)$.

The effect of impaired glucose tolerance on acute pancreatitis has not been fully elucidated [14]. Kikuta et al., in a study conducted in 2015 to show the role of impaired glucose tolerance in the prognosis of acute pancreatitis, it was observed that the mortality rates of Diabetes Mellitus (DM) patients diagnosed with acute pancreatitis were higher than those without. It was predicted that impaired glucose tolerance might be useful in the severity of acute pancreatitis [15]. There is no study in the literature regarding the effect of glucose levels in the emergency department on 1-year mortality in patients diagnosed with acute pancreatitis. In our study, a statistically significant relationship was found between high glucose levels and 1 -year mortality in patients diagnosed with acute pancreatitis $(p<0.001)$. In this respect, our study is original in terms of blood glucose levels and long-term mortality assessment in patients with acute pancreatitis.

In acute pancreatitis, hemoconcentration occurs with a fluid loss to the third space, and accordingly, HCT elevation is observed, and this is an indicator of poor prognosis for acute pancreatitis [16]. Remes-Troche et al., in a study conducted in 2005, it was shown that HCT is a poor predictor of acute pancreatitis prognosis [17]. From the recent studies on the effect of HCT levels on mortality in patients diagnosed with acute pancreatitis, Zhou et al. and Jino et al. in 2019 draw attention $[13,18]$. In these studies, a statistical significance was found between high HCT and BUN levels and mortality. In our study, a significant relationship was found between high urea levels and mortality, supporting previous studies $(p<0.001)$. However, in our study, contrary to these studies, low HCT levels were found to be associated with increased mortality $(p=0.041)$. This may be due to the heterogeneity of the patients included in the studies.

CRP is one of the acute phase reactants synthesized in the liver in response to interleukin-1 and interleukin6 after an inflammatory stimulus. As a result of meta-analysis, 24-48 of CRP in acute pancreatitis. Although it is known that the levels measured per hour have a higher rate of accuracy than the levels seen during the first application to the emergency department in terms of mortality, it is thought that the blood values 
checked at the first application are also significant in terms of mortality [19]. In our study, by the literature, we observed a significant relationship in terms of one-year mortality with CRP levels obtained during the first admission to the emergency department $(\mathrm{p}=0.002)$.

Potassium levels play an essential role in cell membrane electrophysiology, and abnormalities in their levels can lead to malignant arrhythmias [20]. According to the results obtained after meta-analyses, high potassium levels in patients are the only risk factors for mortality, and end-stage renal failure in the subsequent periods [21]. There is no study in the literature evaluating potassium levels and subsequent mortality in patients with acute pancreatitis. In our study, a significant relationship was found with high potassium levels in terms of one-year mortality. The reason for this may be fluid loss in the third space and tissue necrosis observed in acute pancreatitis.

Albumin is an essential modulator of plasma oncotic pressure and an essential transporter of endogenous and exogenous ligands. It is also an important indicator of nutritional status [22]. In a recent study conducted by Hong et al. In 2017, which included 700 patients, low albumin levels in patients with acute pancreatitis were associated with persistent organ failure alone [23]. In our study, a statistically significant result was found between low albumin levels in blood values obtained at first admission and one-year mortality in patients diagnosed with acute pancreatitis in the emergency department $(\mathrm{p}<0.001)$. Our study supports the literature in this respect.

Several studies in the literature draw attention to the relationship between the blood tests obtained at the first admission in patients diagnosed with acute pancreatitis in the emergency department with hospitalization duration and the need for intensive care. In the study by O'Connell et al., 185 patients were included, a statistical significance was observed between RDW and NLR and the duration of hospital stay [24]. In this study, the average length of stay in hospital was 7.65 days, the need for ICU was $8.1 \%$, and mortality was $1.08 \%$. In our study, the average duration of hospital stay was four days, the need for ICU was $3.2 \%$, and the mortality was $8.2 \%$. However, this study was based on 40 -day values in terms of mortality. However, similar to the study by O'Connell et al., In our study, a statistically significant relationship was found between RDW and NLR and the duration of hospital stay. In this respect, our work is compatible with the work of O'Connell et al.

In the study conducted by Azab et al. in 2011, NLR's effect on intensive care admission and hospital stay duration was examined [25]. In this study, the patients were divided into three groups. The relationship between intensive care admissions and the number of days of hospitalization with NLR was evaluated, and statistically significant results were found in both cases. Although the patients were not divided into groups in our study, similar results were obtained with the study by Azab et al. A significant relationship was found between increased NLR in ICU admissions and hospital stay length.

Our study found a statistically significant relationship between high glucose and CRP levels in blood tests taken at the first admission and hospitalization duration and the ICU's need. Also, we observed that low albumin levels and increased PLR correlated with the need for ICU admission. There is no study evaluating the relationship of these values with ICU admissions and length of hospital stay. However, as mentioned above, each of PLR, glucose, and CRP is essential in acute pancreatitis [13-15,19,22,23]. Therefore, we think that PLR, glucose, and CRP are associated with hospital stay length and ICU admissions.

Many studies have tried to calculate cut-off values by dividing patient populations into groups. In our study, the ROC curve was used to calculate the cut-off value. Supiah et al., similarly, in their study in 2013, the ROC curve was used instead of groups to calculate the cut-off value [26]. In this study, the relationship between NLR and severe acute pancreatitis was evaluated, and the cut-off value for NLR at first admission was found to be 10.6. Zhou et al., in their study, patients were divided into three groups and evaluated by looking at 28 -day mortality. In this study, the total mortality rate was found to be $3.45 \%$, the cut-off value for NLR was 12.19, and for PLR, it was 148.85 [13]. In our study, 1-year mortality was evaluated, and when the cut-off value for NLR was 9.09 and 215 for PLR, the mortality rate was found to be $8.1 \%$. There is no other study examining first-admission NLR and PLR cut-off values and 1-year mortality in acute pancreatitis. More studies are needed to determine a more reliable value in cut-off values.

\section{Limitations}

Our study is a single-center retrospective study. Repeating it with more extensive multi-center studies may be beneficial to obtain more reliable data. Blood tests in different ethnic groups may yield different results since they are performed on patients of a limited ethnic group

\section{Conclusions}

High levels of NLR, PLR, RDW, glucose, CRP, urea, potassium, low albumin and hematocrit values at the first admission in the Emergency Department seem to be associated with increased 1-year mortality in acute pancreatitis. High levels of NLR, RDW, glucose, and CRP at the first admission in the emergency department appear to be associated with an increased hospital stay, increased NLR, PLR, glucose, and CRP values low albumin values in acute pancreatitis. 


\section{Additional Information \\ Disclosures}

Human subjects: Consent was obtained by all participants in this study. University of Health Sciences, Kocaeli Derince Training and Research Hospital Ethics Committee issued approval 2020-29. Animal subjects: All authors have confirmed that this study did not involve animal subjects or tissue. Conflicts of interest: In compliance with the ICMJE uniform disclosure form, all authors declare the following: Payment/services info: All authors have declared that no financial support was received from any organization for the submitted work. Financial relationships: All authors have declared that they have no financial relationships at present or within the previous three years with any organizations that might have an interest in the submitted work. Other relationships: All authors have declared that there are no other relationships or activities that could appear to have influenced the submitted work.

\section{References}

1. Singh VK, Bollen TL, Wu BU, et al.: An assessment of the severity of interstitial pancreatitis . Clin Gastroenterol Hepatol. 2011, 9:1098-1103. 10.1016/j.cgh.2011.08.026

2. van Santvoort HC, Bakker OJ, Bollen TL, et al.: A conservative and minimally invasive approach to necrotizing pancreatitis improves outcome. Gastroenterology. 2011, 141:1254-1263. 10.1053/j.gastro.2011.06.073

3. Windsor JA: Assessment of the severity of acute pancreatitis: no room for complacency . Pancreatology. 2008, 8:105-109. 10.1159/000123604

4. Robert JH, Frossard JL, Mermillod B, et al.: Early prediction of acute pancreatitis: prospective study comparing computed tomography scans, ranson, Glascow, acute physiology and chronic health evaluation II scores, and various serum markers. World J Surg. 2002, 26:612-619. 10.1007/s00268-001-0278-y

5. Staubli SM, Oertli D, Nebiker CA: Laboratory markers predicting severity of acute pancreatitis . Crit Rev Clin Lab Sci. 2015, 52:273-283. 10.3109/10408363.2015.1051659

6. Gao W, Yang HX, Ma CE: The value of BISAP score for predicting mortality and severity in acute pancreatitis: a systematic review and meta-analysis. PLoS One. 2015, 10:0142025. 10.1371/journal.pone.0130412

7. Triester SL, Kowdley KV: Prognostic factors in acute pancreatitis . J Clin Gastroenterol. 2002, 34:167-176. 10.1097/00004836-200202000-00014

8. Khanna AK, Meher S, Prakash S, et al.: Comparison of Ranson, Glasgow, MOSS, SIRS, BISAP, APACHE-II, ctsi scores, IL-6, CRP, and procalcitonin in predicting severity, organ failure, pancreatic necrosis, and mortality in acute pancreatitis. HPB Surg. 2013, 2013:367581. 10.1155/2013/367581

9. Zheng J, Cai J, Li H, et al.: Neutrophil to lymphocyte ratio and platelet to lymphocyte ratio as prognostic predictors for hepatocellular carcinoma patients with various treatments: a meta-analysis and systematic review. Cell Physiol Biochem. 2017, 44:967-981. 10.1159/000485396

10. Hazra N, Gulliford M: Evaluating pancreatitis in primary care: a population-based cohort study . Br J Gen Pract. 2014, 64:295-301. 10.3399/bjgp14X679732

11. Spanier B, Bruno MJ, Dijkgraaf MG: Incidence and mortality of acute and chronic pancreatitis in the Netherlands: a nationwide record-linked cohort study for the years 1995-2005. World J Gastroenterol. 2013, 19:3018-3026. 10.3748/wjg.v19.i20.3018

12. Peery AF, Crockett SD, Barritt AS, et al.: Burden of gastrointestinal, liver, and pancreatic diseases in the United States. Gastroenterology. 2015, 149:1731-1741. 10.1053/j.gastro.2015.08.045

13. Zhou H, Mei X, He X, et al.: Severity stratification and prognostic prediction of patients with acute pancreatitis at early phase: A retrospective study. Medicine. 2019, 98:15275-10. 10.1097/MD.0000000000015275

14. Solanki NS, Barreto SG, Saccone GT: Acute pancreatitis due to diabetes: the role of hyperglycaemia and insulin resistance. Pancreatology. 2012, 12:234-239. 10.1016/j.pan.2012.01.003

15. Kikuta K, Masamune A, Shimosegawa T: Impaired glucose tolerance in acute pancreatitis . World J Gastroenterol. 2015, 21:7367-7374. 10.3748/wig.v21.i24.7367

16. Muddana V, Whitcomb DC, Khalid A, et al.: Elevated serum creatinine as a marker of pancreatic necrosis in acute pancreatitis. Am J Gastroenterol. 2009, 104:164-170. 10.1038/ajg.2008.66

17. Remes-Troche JM, Duarte-Rojo A, Morales G, et al.: Hemoconcentration is a poor predictor of severity in acute pancreatitis. World J Gastroenterol. 2005, 11:7018-7023. 10.3748/wjg.v11.i44.7018

18. Jinno N, Hori Y, Naitoh I, et al.: Predictive factors for the mortality of acute pancreatitis on admission . PLoS One. 2019, 10.1371/journal.pone.0221468

19. Staubli SM, Oertli D, Nebiker CA: Laboratory markers predicting severity of acute pancreatitis . Crit Rev Clin Lab Sci. 2015, 52:273-283. 10.3109/10408363.2015.1051659

20. Hoppe LK, Muhlack DC, Koenig W, et al.: Association of abnormal serum potassium levels with arrhythmias and cardiovascular mortality: a systematic review and meta-analysis of observational studies. Cardiovasc Drugs Ther. 2018, 32:197-212. 10.1007/s10557-018-6783-0

21. Kovesdy CP, Matsushita K, Sang Y, et al.: Serum potassium and adverse outcomes across the range of kidney function: a CKD prognosis consortium meta-analysis. Eur Heart J. 2018, 39:1535-1542. 10.1093/eurheartj/ehy100

22. Yuwen P, Chen W, Lv H, et al.: Albumin and surgical site infection risk in orthopaedics: a meta-analysis . BMC Surg. 2017, 17:10.1186/s12893-016-0186-6

23. Hong W, Lin S, Zippi M, et al.: Serum albumin is independently associated with persistent organ failure in acute pancreatitis. Can J Gastroenterol Hepatol. 2017, 2017:5297143-10. 10.1155/2017/5297143

24. O'Connell RM, Boland MR, O'Driscoll J, et al.: Red cell distribution width and neutrophil to lymphocyte ratio as predictors of outcomes in acute pancreatitis: a retrospective cohort study. Int J Surg. 2018, 55:124-127. 10.1016/j.ijsu.2018.05.028 


\section{Cureus}

25. Azab B, Jaglall N, Atallah JP, et al.: Neutrophil-lymphocyte ratio as a predictor of adverse outcomes of acute pancreatitis. Pancreatology. 2011, 11:445-452. 10.1159/000331494

26. Suppiah A, Malde D, Arab T, et al.: The prognostic value of the neutrophil-lymphocyte ratio (NLR) in acute pancreatitis: identification of an optimal NLR. J Gastrointest Surg. 2013, 17:675-681. 10.1007/s11605-0122121-1 